\title{
Quality of life of children with spinal muscular atrophy and their caregivers from the perspective of caregivers: a Chinese cross-sectional study
}

\author{
Mei Yao ${ }^{1 \dagger}$, Ying Ma ${ }^{1 \dagger}$, Ruiying Qian ${ }^{1}$, Yu Xia ${ }^{1}$, Changzheng Yuan², Guannan Bai ${ }^{1 *}$ and Shanshan Mao ${ }^{1 *}$ (D)
}

\begin{abstract}
Background: Spinal muscular atrophy (SMA) is an autosomal-recessive motor neuron disease leading to dysfunction of multiple organs. SMA can impair the quality of life (QoL) of patients and family. We aimed to evaluate the QoL of children with SMA and their caregivers and to identify the factors associated with QoL in a cross-sectional study conducted in China.

Methods: We recruited 101 children aged 0-17 years with SMA and their caregivers from a children's hospital in China. Twenty-six children had type I SMA, 56 type II and 19 type III. Each child's QoL was measured by the Pediatric Quality of Life Inventory 3.0 Neuromuscular Module (PedsQL NMM), which was completed by the child's caregivers. The caregiver's QoL was measured by the Pediatric Quality of Life Inventory Family Impact Module (PedsQL FIM). Information on sociodemographic characteristics, disease-specific characteristics, and treatments were collected using the proxy-reported questionnaire. Two-sample $t$ tests and one-way ANOVA were used to compare differences in average scores of QoL across subgroups.

Results: Children with type III SMA had a higher average Total score of PedsQL NMM and higher average scores in domains Neuromuscular disease and Family resources than children with type I or type II SMA $(p<0.001)$. Caregivers of children with type III SMA reported higher average scores in the domains of Physical, Emotional, Social, and Cognitive functioning of the PedsQL FIM than those of children with types I or II SMA $(p<0.05)$. In addition, disease-related characteristics (e.g. limited mobility, stable course of disease, skeleton deformity, and digestive system dysfunction) and respiratory support were associated with lower average scores of PedsQL NMM and PedsQL FIM $(p<0.05)$. Exercise training, multidisciplinary team management and use of the medication Nusinersen were each associated with higher average scores in both PedsQL NMM and FIM $(p<0.05)$.
\end{abstract}

Conclusion: Our study has demonstrated factors that may impair or improve QoL of children patients with SMA and their parents. Particularly, QoL was relatively poor in children with type I and type II SMA as well as in their caregivers compared to those with type III SMA. We strongly recommend that standard of care in a multidisciplinary team be strengthened to improve the QoL of SMA patients. Our study called for increased attention from clinical physicians

\footnotetext{
*Correspondence: baiguannan@gmail.com; 6307003@zju.edu.cn

${ }^{\dagger}$ Mei Yao, Ying Ma have contributed equally for this article.

1 Department of Neurology, Children's Hospital, Zhejiang University

School of Medicine, National Clinical Research Center for Child Health, Hangzhou 310052, China

Full list of author information is available at the end of the article
}

(c) The Author(s) 2021. Open Access This article is licensed under a Creative Commons Attribution 4.0 International License, which permits use, sharing, adaptation, distribution and reproduction in any medium or format, as long as you give appropriate credit to the original author(s) and the source, provide a link to the Creative Commons licence, and indicate if changes were made. The images or other third party material in this article are included in the article's Creative Commons licence, unless indicated otherwise in a credit line to the material. If material is not included in the article's Creative Commons licence and your intended use is not permitted by statutory regulation or exceeds the permitted use, you will need to obtain permission directly from the copyright holder. To view a copy of this licence, visit http://creativecommons.org/licenses/by/4.0/. The Creative Commons Public Domain Dedication waiver (http://creativeco mmons.org/publicdomain/zero/1.0/) applies to the data made available in this article, unless otherwise stated in a credit line to the data. 
on measuring QoL in their clinical practices in order to enhance the understanding of impacts of SMA and to make better decisions regarding treatment.

Keywords: Spinal muscular atrophy, Quality of life, Disease-related characteristic, Medical intervention, Proxy-report

\section{Introduction}

Spinal muscular atrophy (SMA) is a rare, autosomalrecessive neuromuscular disease caused by genetic mutation of the survival motor neuron (SMN) 1 gene on chromosome $5 \mathrm{q} 13$. This mutation results in reduced levels of the SMN protein, causing muscle weakness and atrophy [1-5]. SMA is traditionally divided into five clinical subtypes (type 0, I, II, III, IV) based on the age of onset of symptoms and on the highest achieved motor-function milestones [6,7]. Type 0 is the most severe subtype that onsets during the prenatal period and survives less than one month after birth. Type I is the most common subtype in living patients; it usually presents before 6 months of age. Non-sitter always refer to type I patients who were never learn to sit up independently, and their life expectancy is seldom more than 2 years without respiratory support. Type II usually onsets between 6 and 18 months of age. Sitter was defined as who can sit independently but are never be able to walk. Type II patients usually can live to adulthood. Symptoms of type III normally present after age 18 months; walker refer to some patients can acquire independent ambulation, although some may lose the ability to walk in adulthood owing to the progressive nature of the disease. Life spans of these patients are almost identical to those of the general population. Type IV is the rarest, has the lowest morbidity and mortality, and occurs after 20 years of age. Life span of type IV patients is similar to that of type III patients [8, 9].

In addition to causing damage of motor neurons, SMN protein deficiency is also detrimental to the functioning of multiple tissues including those found in skeletal muscle, heart, autonomic and enteric nervous systems, metabolic/endocrine system, lymphatic systems, and reproductive system [10-12]. Therefore, dysfunction of multiple organ systems may occur in a SMA patient as the disease progresses. SMA patients may need medical care and nursing support for daily activities as well as for long-term co-management of several medical devices $[13,14]$. Current treatment options in SMA include (1) ventilatory support including noninvasive and invasive ventilation, (2) management of secretions including inhalational therapies and section, (3) feeding support including nasal feeding or placement of a percutaneous endoscopid gastrostomy and (4) supply with medical devices and orthosis [15-17].
Improvements regarding survival and QoL of SMA patients could be achieved by these options [7].

QoL is a multidimensional concept and is an important patient-reported outcome measure in clinical research and practice. According to the World Health Organization, QoL is defined as "an individual's perception of their position in the life in the context of the culture in which they live and in relation to their goals, expectations, standards and concerns" [18]. While investigating QoL in the clinical setting, it is important to understand the patient's experience, to evaluate the effectiveness of treatments, and to optimize individual therapy plans, which ultimately improve the patient's well-being $[19,20]$.

In pediatric neurology research, attentions mostly has focused on developing novel technologies and pharmaceuticals for children with SMA. Few studies have focused on investigating the impacts of these clinical treatments on the QoL of patients and caregivers. One study conducted in Europe by Rouault et al. described the disease impact on general well-being and therapeutic expectations of SMA type II and III patients [21]. This study highlighted the patient's perspective in terms of treatment and living with SMA [21]. However, the study used a self-developed questionnaire based on expert opinion instead of a validated survey instrument for measuring QoL. Based on a literature review, we found that the PedsQL 3.0 Neuromuscular Module (PedsQL NMM) can be used to estimate the QoL of SMA patients, whereas the PedsQL Family Impact Module (PedsQL FIM) can be used as a supplement of the PedsQL NMM to reflect the caregivers' QoL and the family impact. Weaver et al. found that PedQL FIM captured significant differences in functioning domains of QoL including physical, emotional, social, and family relations between SMA Type I and II [22]. In addition, this study demonstrated significant differences in the communication domain of the proxy-reported PedsQL NMM [22]. Nusinersen treatment did not impact proxy-reported QoL, whereas gastrostomy tube and ventilation support decreased children's QoL [22]. The analysis of parents' questionnaires revealed that different types of SMA and clinical treatment can significantly affect the QoL of SMA patients. Published studies of the QoL of SMA patients and caregivers have been conducted in the United States or in Europe. To our best knowledge, no similar study has been conducted in China. Therefore, in the present study we aimed to evaluate the QoL of children with SMA and 
their caregivers and to identify the factors associated with QoL (such as SMA subtypes, disease-related characteristics and treatments) for patients and caregivers that participated in a hospital-based, cross-sectional study in China.

\section{Methods}

\section{Participants and design}

During 16-28 March 2020 we conducted a questionnaire-based survey of 101 caregivers, which included the parents or grandparents of SMA patients recruited from the Department of Neurology of the Children's Hospital of Zhejiang University School of Medicine in Hangzhou, China. The inclusion criteria for caregivers were (1) their child was diagnosed as SMA by genetic testing and (2) their child's age ranged from 0 to 18 years. Caregivers were excluded if they could not understand or complete the questionnaire correctly. In accordance with the principle of voluntary participation, the study questionnaire was sent by website to the caregivers with detailed information about the purpose and methods of this study. All eligible participants provided signed, informed consent before they completed the questionnaire, and the study was approved by the Ethics Committee of the Children's Hospital of Zhejiang University School of Medicine (2019-IRB-171).

Before the questionnaire was used, we conducted a preliminary test of the feasibility, comprehensibility, and acceptability of the questions (survey items) and their multiple-choice answers. During this test 25 questionnaires were sent out, 24 were received yielding an effective recovery of $96 \%$. The results of the preliminary test showed that the population response rate was very high and that our investigation was feasible. It took the caregiver 20-30 min to fill in the questionnaire, so we set the recommended length of time of completion of questionnaire as $35 \mathrm{~min}$ in the instruction texts of the final survey questionnaire. During the survey of all participants, the data were collected by trained physicians using a one-to-one internet survey. After logging onto the questionnaire website, all caregivers were asked to complete the PedsQL FIM section. In addition, the caregivers of SMA patients aged 5 years and older were asked to complete the PedsQL NMM section. All caregivers immediately submitted their answers upon completion of the questionnaire.

\section{QoL measures}

PedsQL NMM and PedsQL FIM were used to measure QoL of patients with SMA and their caregivers. In addition, the questionnaire included some questions to collect the information on socio-demographic characteristics, disease-related characteristics, and medical interventions/treatment plan of the patients. Several terms were used in these additional questions. For example, a non-Sitter was defined as a patient who was unable to sit. A sitter was defined as a patient who could sit without aid but could not walk alone, whereas a patient who could walk alone was defined as walker. Skeletal dysfunctions were defined as structural changes in the skeletal system that result in functional changes, such as scoliosis, hip dislocation, tendon contracture, joint deformation, etc. Digestive system dysfunctions were defined as disordered functions of digestive organs including the gastrointestinal tract, the liver and biliary system, and the pancreas. The main symptoms of digestive system dysfunctions included salivation, difficulty swallowing, constipation, etc. Stable course of disease was defined as a state of disease progression wherein patient's athletic ability and intensity keep no progressive levels during past 6 months. For example, a patient who was 9 months old but was unable to hold her/his head up steadily from 3 months old. Exercise training was defined as rehabilitation training in professional institutions. A patient that had not accepted any medical service was defined as nomedical service (N-MS). A patient that only accepted a neurology doctor but was not under multidisciplinary team (MDT) was defined as medical service (MS). A patient that accepted MDT was defined as accepted standard of care (SOC) in a MDT management program.

We used PedsQL NMM, a proxy-reported, 25-item survey instrument, to measure health-related QoL in children with neuromuscular disorders. This instrument contains three dimensions: About My Child's Neuromuscular Disease (17 items, with emphasis on physical functioning), Communication (three items), and About Our Family Resources (five items). For each item, caregivers were asked to rate the influence of a certain problem in the past month. Each multiple-choice answer was scored from 0 (never a problem) to 4 (almost always a problem). Item scores were reversed and linearly transformed to a 0 to 100 scale $(0=100,1=75,2=50,3=25$, and $4=0)$; therefore, a higher scores indicated a higher QoL. PedsQL NMN is a reliable survey instrument, and Cronbach's alpha for each dimension exceeds 0.70 [23].

The PedsQL FIM was designed to assess the impact of pediatric chronic health conditions on caregivers' QoL and family functions in the past month. The PedsQL FIM includes the following six scales of a caregiver's selfreported functioning: physical functioning (six items), emotional functioning (five items), social functioning (four items), cognitive functioning (five items), communication (three items), and worry (five items). In addition, the PedsQL FIM explored the following two scales of family functioning reported by the primary caregiver: daily activities (three items) and family relationships (five 
items). Each item included a five-point, multiple-choice response ranging from 0 (never a problem) to 4 (always a problem). These item scores were reversely graded and linearly transformed to a $0-100$ scale $(0=100,1=75$, $2=50,3=25,4=0$ ); therefore, higher scores indicated better functioning or less negative impacts. Initial validation studies indicated that PedsQL FIM has a relatively high internal consistency with Cronbach's alpha values exceeding 0.70 [24].

\section{Statistical analysis}

Descriptive analysis was applied to describe the characteristics of the study population. Continuous measurements were summarized as means \pm standard deviations (SDs), and categorical measurements were summarized using frequencies with percentages. A normal distribution of our data was tested using the normality test and distribution curve. A two-sample t-test was used to compare the difference in average scores of QoL between two subgroups; whereas a one-way ANOVA was used to compare average scores of QoL among three or more subgroups. In addition, we used Cohen's effect size (Cohen's $d$ ) to assess the clinical relevance in terms of pairwise differences in average score between groups (including differences between subtypes of SMA, disease-related characteristics, and acceptance categories of clinical treatments). Cohen's $d$ was calculated as the absolute value of the difference in average scores divided by the largest SD and was interpreted as follows: $0.2 \leq d<0.5$, small difference; $0.5 \leq d<0.8$, moderate difference; and $d \geq 0.8$, large difference [25].

All statistical tests were two-tailed and their significance was indicated by $p<0.05$. All the analyses were conducted using SPSS version 22 (IBM Corp. NY, USA).

\section{Results}

\section{Characteristics of the study population}

Socio-demographics characteristics, disease-related characteristics and medical interventions/treatment plan of the patients in our study are summarized in Table 1. A total of 101 caregivers with SMA children participated in our study. All caregivers completed the PedsQL FIM questionnaire; however, only 87 caregivers completed the PedsQL NMM questionnaire because this survey instrument applies only to children of ages $2-18$ years. Ten patients depended on invasive ventilation, 23 patients used a suction tube and only 1 child received a non-invasive ventilator treatment. Sixteen patients were treated by special formula feeding, 2 patients used nasal feeding tube and 1 child received gastrostomy in nutritional support.
Table 1 The demographics characteristics of SMA patients $(n=101)$

\begin{tabular}{|c|c|}
\hline Variable & $N(\%)$ \\
\hline \multicolumn{2}{|l|}{ Child sex } \\
\hline Male & $49(48.50)$ \\
\hline Female & $52(51.50)$ \\
\hline \multicolumn{2}{|l|}{ Child primary diagnosis } \\
\hline SMA type I & $26(25.70)$ \\
\hline SMA type II & $56(55.40)$ \\
\hline SMA type III & $19(18.80)$ \\
\hline Average age of SMA type (years) & Age range of SMA type (years) \\
\hline SMA type l: 5.28 & $0.50-16.17$ \\
\hline SMA type II: 6.90 & $0.92-16.00$ \\
\hline SMA type III: 9.35 & $2.08-13.67$ \\
\hline Disease duration & Age range of disease duration (years) \\
\hline SMA type I & $4.86(0.33-15.58)$ \\
\hline SMA type II & $6.12(0.17-15.50)$ \\
\hline SMA type III & $5.9(1.00-13.67)$ \\
\hline \multicolumn{2}{|l|}{ Mobility } \\
\hline Non-sitter & $46(45.50)$ \\
\hline Sitter & $39(38.60)$ \\
\hline Walker & $16(15.80)$ \\
\hline \multicolumn{2}{|l|}{ Stable course of disease } \\
\hline No & $41(40.60)$ \\
\hline Yes & $60(59.40)$ \\
\hline \multicolumn{2}{|l|}{ Skeletal deformity } \\
\hline No & $27(26.70)$ \\
\hline Yes & $74(73.30)$ \\
\hline \multicolumn{2}{|l|}{ Digestive system dysfunction } \\
\hline No & $78(77.20)$ \\
\hline Yes & $23(22.80)$ \\
\hline \multicolumn{2}{|l|}{ Medical services } \\
\hline N-MS & $67(66.30)$ \\
\hline MS but not U-MDT & $21(20.80)$ \\
\hline U-MDT & $13(12.90)$ \\
\hline \multicolumn{2}{|l|}{ Exercise training } \\
\hline No & $56(55.40)$ \\
\hline Yes & $45(44.60)$ \\
\hline \multicolumn{2}{|l|}{ Respiratory support } \\
\hline No & $75(74.30)$ \\
\hline Yes & $26(25.70)$ \\
\hline \multicolumn{2}{|l|}{ Nutritional support } \\
\hline No & $82(81.20)$ \\
\hline Yes & $19(18.80)$ \\
\hline \multicolumn{2}{|l|}{ Scoliosis surgery } \\
\hline No & $99(98.00)$ \\
\hline Yes & $2(2.00)$ \\
\hline \multicolumn{2}{|l|}{ Nusinersen treatment } \\
\hline No & $92(91.10)$ \\
\hline Yes & $9(8.90)$ \\
\hline
\end{tabular}

SMA spinal muscular atrophy, N-MS no medical services, MS but not, U-MDT medical services but not under multidisciplinary team, U-MDT Under multidisciplinary team 
Differences in the average scores of PedsQL NMM across SMA subtypes

The average scores of PedsQL NMM stratified by SMA subtypes are summarized in Table 2. The average total score and average scores of domains Neuromuscular diseases and Family resources were significantly higher in patients with SMA type III than in those with SMA type I $(64.89$ vs. $43.63, p<0.001, d=1.08 ; 65.32$ vs. $40.53, p<0.001, d=1.23$; and 58.68 vs. $38.75, p=0.006$, $d=0.74)$. Similarly, the average total score and average scores of domains Neuromuscular diseases and Family resources were significantly higher in patients with SMA type III than in those with SMA type II $(64.89$ vs. $48.79, p<0.001, d=0.82 ; 65.32$ vs. $49.10, p<0.001$, and 58.68 vs. $33.94, p<0.001, d=0.92$ ). In contrast, no statistically significant differences were found among SMA subtypes in the average score of domain Communication.

The average scores of PedsQL FIM stratified by subtype are summarized in Table 3 . The average total score and the average scores of domains Physical, Emotional, Social, Cognitive functioning were significantly higher in patients with SMA type III than in those with SMA types I or II $(p<0.05)$. The largest effect size was seen in the average scores of domain Physical functioning when comparing patients of SMA types III and I $(d=1.04)$. No statistically significant differences were found in the above-mentioned average scores between patients of SMA types I and II $(p>0.05)$.

Table 2 Average scores of proxy-reported PedsQL 3.0 Neuromuscular Module across SMA subtypes $(n=87)$

\begin{tabular}{|c|c|c|c|c|c|c|}
\hline \multirow{2}{*}{$\begin{array}{l}\text { Children's QoL accessed } \\
\text { from the caregiver }\end{array}$} & \multirow{2}{*}{$\begin{array}{l}\text { SMA Type I } \\
\text { Mean } \pm \text { SD }(n=16)\end{array}$} & \multirow{2}{*}{$\begin{array}{l}\text { SMA Type II } \\
\text { Mean } \pm \text { SD }(n=52)\end{array}$} & \multirow{2}{*}{$\begin{array}{l}\text { SMA Type III } \\
\text { Mean } \pm \text { SD } \\
(n=19)\end{array}$} & \multicolumn{3}{|c|}{ Effect size (d) } \\
\hline & & & & | versus || & I versus III & II versus III \\
\hline Total score & $43.63 \pm 13.08$ & $48.79 \pm 13.04$ & $64.89 \pm 19.75^{b, c}$ & 0.39 & 1.08 & 0.82 \\
\hline Neuromuscular disease & $40.53 \pm 13.86$ & $49.10 \pm 13.65$ & $65.32 \pm 20.21^{b, c}$ & 0.62 & 1.23 & 0.80 \\
\hline Communication & $69.27 \pm 33.85$ & $71.79 \pm 26.72$ & $74.56 \pm 27.70$ & 0.07 & 0.16 & 0.10 \\
\hline Family resources & $38.75 \pm 13.60$ & $33.94 \pm 20.28$ & $58.68 \pm 26.87^{b, c}$ & 0.24 & 0.74 & 0.92 \\
\hline
\end{tabular}

Effect size (Cohen's $d$ ) is interpreted as: $0.2 \leq d<0.5$ small difference, $0.5 \leq d<0.8$ moderate, and $d \geq 0.8$ large

$S M A$ spinal muscular atrophy

Bold prints indicate $P<0.05$

a Post hoc significance between type I and type II

b Post hoc significance between type I and type III

c Post hoc significance between type II and type III

Table 3 Average scores of PedsQL Family Impact Module across SMA subtypes $(n=101)$

\begin{tabular}{|c|c|c|c|c|c|c|}
\hline \multirow[t]{2}{*}{ Caregivers' quality of life } & \multirow{2}{*}{$\begin{array}{l}\text { SMA Type I } \\
\text { Mean } \pm \text { SD }(n=26)\end{array}$} & \multirow{2}{*}{$\begin{array}{l}\text { SMA Type II } \\
\text { Mean } \pm \text { SD }(n=56)\end{array}$} & \multirow{2}{*}{$\begin{array}{l}\text { SMA Type III } \\
\text { Mean } \pm \text { SD }(n=19)\end{array}$} & \multicolumn{3}{|c|}{ Effect size (d) } \\
\hline & & & & I versus II & I versus III & II versus III \\
\hline Total score & $34.97 \pm 21.97$ & $40.56 \pm 17.76$ & $53.80 \pm 21.87^{b, c}$ & 0.25 & 0.86 & 1.45 \\
\hline Physical functioning & $29.01 \pm 25.18$ & $45.16 \pm 21.52$ & $59.43 \pm 29.23^{a, b, c}$ & 0.64 & 1.04 & 0.49 \\
\hline Emotional functioning & $31.54 \pm 28.42$ & $33.93 \pm 20.64$ & $54.47 \pm 23.27^{b, c}$ & 0.08 & 0.81 & 0.88 \\
\hline Social functioning & $29.33 \pm 26.15$ & $37.28 \pm 25.11$ & $55.59 \pm 29.16^{b, c}$ & 0.30 & 0.90 & 0.63 \\
\hline Cognitive functioning & $40.00 \pm 24.78$ & $45.18 \pm 24.77$ & $61.58 \pm 25.71^{b, c}$ & 0.21 & 0.84 & 0.64 \\
\hline Communication & $41.99 \pm 31.49$ & $43.75 \pm 25.69$ & $55.26 \pm 31.21$ & 0.06 & 0.42 & 0.37 \\
\hline Worry & $30.00 \pm 26.87$ & $26.16 \pm 17.94$ & $37.63 \pm 27.35$ & 0.14 & 0.28 & 0.42 \\
\hline Daily activities & $28.20 \pm 25.94$ & $36.61 \pm 24.40$ & $40.79 \pm 24.52$ & 0.32 & 0.49 & 0.17 \\
\hline Family relationships & $49.81 \pm 28.02$ & $54.46 \pm 21.78$ & $60.26 \pm 23.24$ & 0.17 & 0.37 & 0.25 \\
\hline
\end{tabular}

Effect size (Cohen's $d$ ) is interpreted as: $0.2 \leq d<0.5$ small difference, $0.5 \leq d<0.8$ moderate, and $d \geq 0.8$ large

$S M A$ spinal muscular atrophy

Bold prints indicate $P<0.05$

a Post hoc significance between type I and type II

b Post hoc significance between type I and type III

c Post hoc significance between type II and type III 


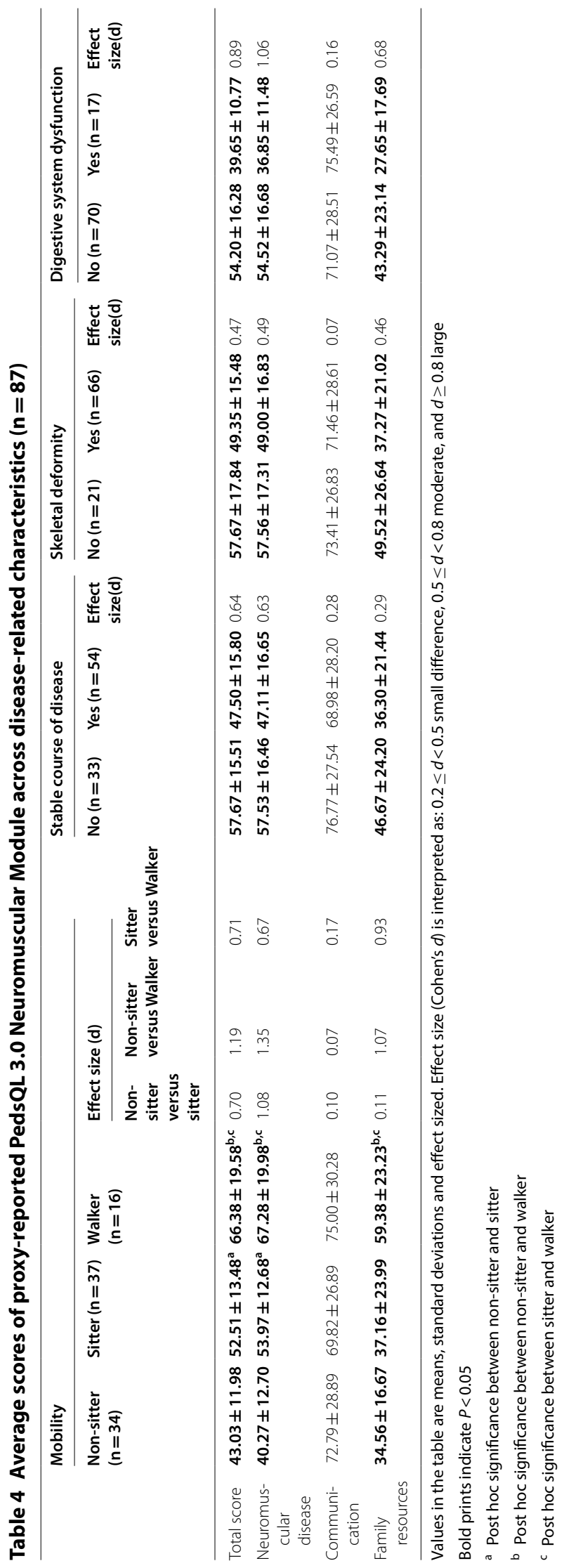




\section{Differences in the average scores of PedsQL NMM across disease-specific characteristics}

The average score of PedsQL NMM stratified by disease-related characteristics are summarized in Table 4. Patients categorized as non-sitter or sitter were reported by their parents to have relatively average scores (Total and domains Neuromuscular disease and Family resources) compared to parents that were able to walk independently $(p<0.05)$. In particular, in comparisons of Non-sitter or Walker, the effect sizes were $1.19,1.35$ and 1.07 for differences in average Total score, average score of domain Neuromuscular disease, and average score of domain Family resources, respectively. The average Total score and the average scores of domains Neuromuscular disease and Family resources were significantly higher in children without stable course of disease than in those with stable course of disease $(p<0.01)$; the effect sizes ranged from 0.29 to 0.64 . The average Total scores and the average scores of domains Neuromuscular disease and Family resources were significantly higher in children without motor skeleton deformity than in those with skeleton deformity $(p<0.05)$; the effect sizes ranged from 0.46 to 0.49 . The average Total scores and the average scores of domains Neuromuscular disease and Family resources were significantly higher among children with normal digestive system function than in those with digestive system dysfunction $(p<0.01)$; the effect sizes ranged from 0.68 to 1.06 .

\section{Differences in the average scores of PedsQL FIM across disease-specific characteristics}

The average scores of PedsQL FIM stratified by disease-related characteristics are summarized in Table 5. Parents with children categorized as Non-sitter or Sitter reported significantly lower average Total scores and average scores of domains Physical, Emotional, Social, Cognitive functioning compared with the same scores of children categorized as Walker $(p<0.05)$; the largest effect size was 1.40 in the comparison between Non-sitter and Walker categories. The average scores of domains Physical and Emotional functioning were significantly lower for children with skeletal deformity than for those without skeletal deformity $(p<0.05)$. Regarding digestive system dysfunction, the average Total score and the average scores of domain Daily activities and domains Physical, Emotional, Social, Cognitive functioning were significantly lower in children with the dysfunction than in those that lacked the dysfunction $(p<0.01)$; the effect sizes ranged from $0.56-1.26$.

\section{Differences in the average scores of PedsQL NMM across the clinical treatments}

The average scores of PedsQL NMM stratified by clinical treatments are summarized in Table 6. The average Total scores and the average scores of domain Neuromuscular disease were significant higher in children that received Exercise training than in those which did not $(p<0.001)$; the effect sizes were 0.60 and 0.75 , respectively. The average Total scores and the average scores of domain Neuromuscular disease were significant lower in children that received Respiratory support than in those which did not $(p<0.001)$; the effect sizes were 0.57 and 0.79 , respectively. Regarding MDT, significant differences in average Total score and in average scores of domains Neuromuscular disease and Family resources were detected between groups with SOC in MDT and groups without MDT (N-MS and MS groups). Children in the MS group had significantly higher average Total scores and average scores in domains Neuromuscular disease and Family resources than children in N-MS group $(p<0.05)$. Children under MDT had significantly higher average Total scores and average scores in domains Neuromuscular disease and Family resources than children in N-MS groups $(p<0.05)$ or those in MS group $(p<0.05)$. Effect sizes ranged from 0.13 to 0.90 .

\section{Discussion}

The present study assessed proxy-reported quality of life of children with SMA and self-reported quality of life of their caregivers in China. We found that quality of life of patients and caregivers differed across SMA subtype, disease-related characteristics, and treatment characteristics.

We found average scores were higher in terms of the overall quality of life score, Neuromuscular diseases domain, and Family resource domain of PedsQL NMM of patients with type III compared with those in patients with type II or type I, which was consistent with previous studyhaving the lowest average score. It could be explained by the difference in severity across subtypes. Additionally, the effect sizes were moderate to large, which indicated that the differences in quality of life of patients had reached the threshold of clinical relevance and were worthy of attention from health professionals. Meaghann et al. found no statistically significant difference across SMA subtypes in the Neuromuscular diseases domain of PedsQL NMM, which was different with our finding [22]. It might be explained by the difference in the severity of SMA between our sample and theirs. Patient's physical function was worse in our sample, especially in patients with type I and II. 


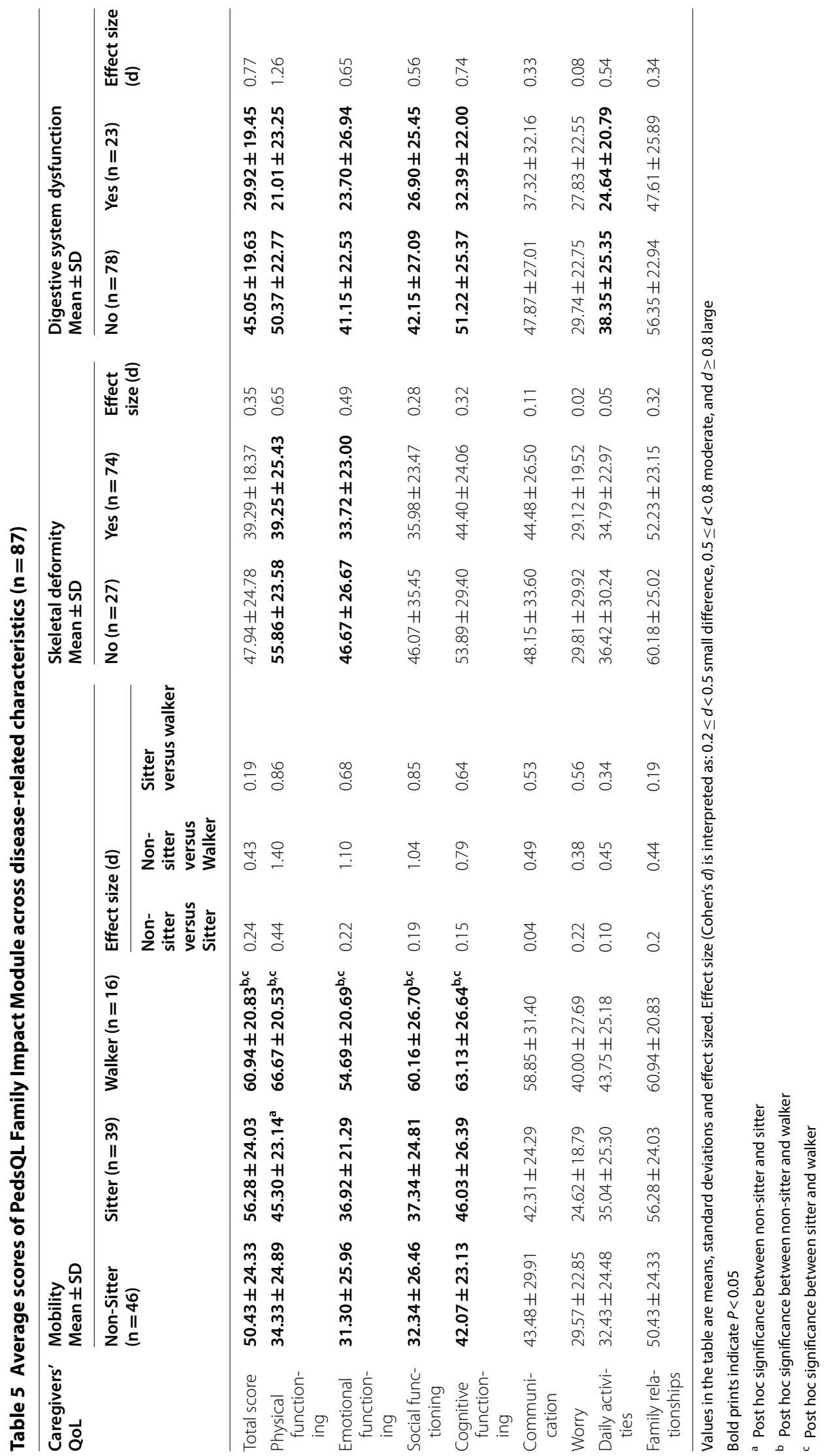




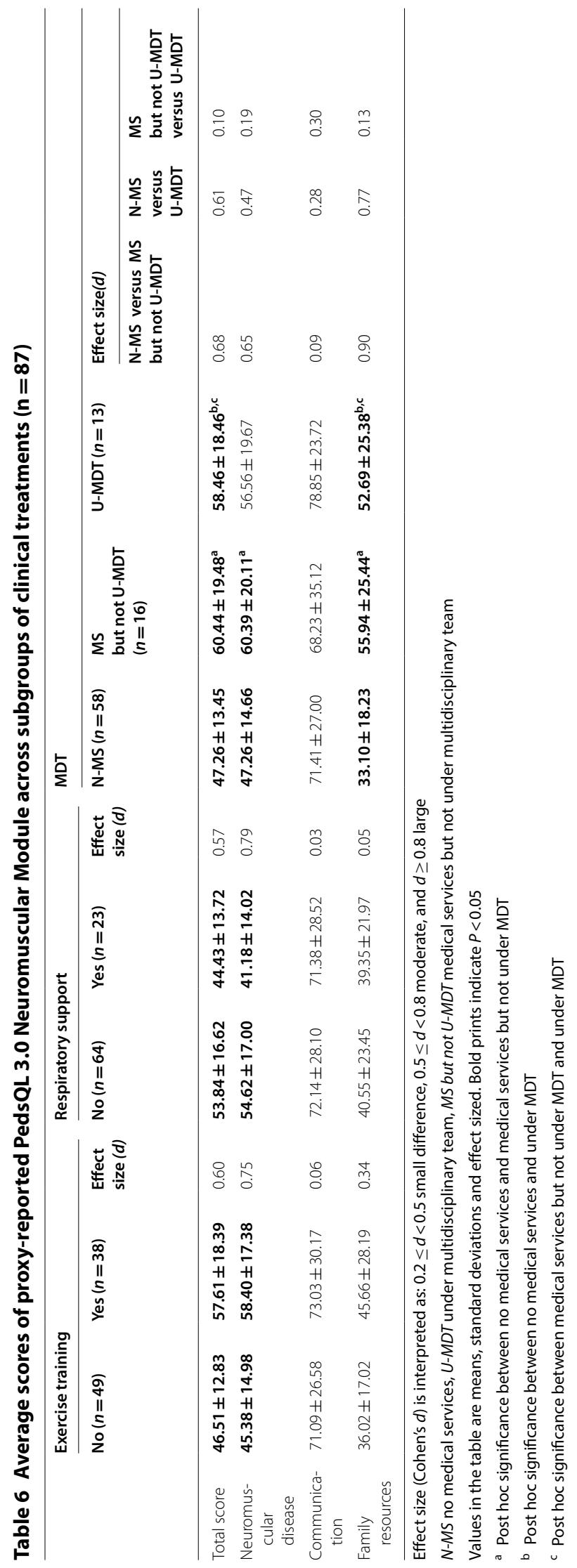


Our study showed better QoL of caregivers of patients with type III than those of patients with type I and II, which was explained by the nature of the three subtypes of SMA. Type III patients were the least severe type. Most patients with type III have the ability to move autonomously, to complete various functional exercises by themselves, and are able to walk alone, which may put less burden on their caregivers, compared with other two types of patients who cannot walk or sit. Our study did not find significant difference across SMA types in the Communication, Worry, Daily activities, or Family relations domains of PedsQL FIM. The potential explanation was that regardless of subtypes, all the caregivers gradually accepted the fact of living together with ill children and adapted to the situation. So the impact of SMA on caregiver's worry level, daily activities and on family communication about patients/disease and family relations may stay stable during treatment phase. That may be the reason why our study did not find the differences in the above-mentioned domains across subtypes.

Disease-related characteristics of patients with SMA include skeletal malformation, digestive dysfunction, stable course of disease, and current motor ability. In the present study, the above-mentioned characteristics of SMA were associated with lower average score in Neuromuscular diseases domain and Family resources domain of PedsQL NMM than patients without these complications. As SMA is a progressive disease, complications such as skeletal malformation, digestive disorders, respiratory disorders, and motor function degeneration may influence patient's QoL gradually $[10,11]$. Our study also supported the findings from a previous study that showed motor function affected multiple aspects of the patient's life [27]. In addition to the statistical significances, the effect sizes regarding Neuromuscular disease and Family resource domain of PedsQL NMM were moderate and large, indicating that disease-related characteristics may impact the QoL of patients, which reached the threshold of clinical relevance and warranted attention from clinical professionals during their practice.

Regarding caregiver's QoL measured by PedsQL FIM, the physical and emotional functions of the caregivers of patients with skeletal malformations were worse compared with caregivers of patients without skeletal malformations. Skeletal malformation usually accompanies with progressive muscular atrophy and muscle weakness that often worsen skeletal malformation in return, which is a circle leading to impaired motor ability overtime [13, 14, 28]. Under this situation, caregivers had to spend lots of time and energy on daily care of patients and had fewer time for themselves, which may lead to great burden on caregiver's physical and mental health. Our study also suggested that digestive system dysfunction may negatively influence caregiver's QoL in multiple domains, such as physical, mental, social and cognitive function. Digestive system dysfunction such as dysphagia and other digestive disorders often appear in the last stage of SMA, which seriously affects the patient's overall health [29]. Meanwhile, caregivers were under great burden to take care of patients, such as financial burden, less personal time and great mental stress, which deteriorated their health and QoL [17].

Our results suggested that clinical treatments may improve the QoL of patients with SMA. Early exercise training is beneficial for remaining or improving the mobile function [30,31]. Early studies showed that exercise training can activate the motor neurons to promote the recovery, and effectively improve the motor function of patients with neuromuscular degeneration disease, including Duchenne muscular dystrophy and SMA, which eventually improved patient's QoL [32-35].

Though previous studies showed that respiratory support could improve patient's health outcomes [22, 36, 37], our study showed that patients with respiratory support had lower average scores and lower score of Neuromuscular disease domain of PedsQL NMM reported by caregivers, compared with those without respiratory support. The reasonable explanation was that patients received respiratory support when their condition deteriorated to a very severe situation. In this case, their overall health status and well-being actually were worse than those who did not receive respiratory support.

Our study showed that the multidisciplinary team (MDT) management was associated with better overall QoL patients compared with patients without MDT management. The effect size was moderate, which indicated a minimally clinically important difference. MDT management refers to having more than two related disciplines in order to implement clinical treatments for specific diseases [38, 39]. As shown in previous studies, MDT management in other chronic diseases, such as tuberous sclerosis complex (TSC), Prader-Willi syndrome, pediatric chronic pancreatitis, pediatric medulloblastoma, and asthma, this approach was conducive to reducing caregiver burden, to decreasing healthcare load and to improve patient's QoL [40-43]. According to our clinical experience, low awareness of MDT health management interventions among caregivers, which may delay early interventions to SMA. Based on our observations and previous studies, we suggested that raise the caregivers' awareness of MDT management, which is beneficial to conduct the early intervention and eventually improve the health outcomes, such as QoL of patients [13, 14].

We did not show the results in tables regarding the differences of quality of life across the subgroup using Nusinersen and not using this medication because only 
six patients in our study received Nusinersen treatment. Briefly, the result showed that patients with Nusinersen treatment was reported higher average score in the Neuromuscular diseases and Family resources domains of PedsQL NMM than those without such treatment. Nusinersen is the first effective medication for SMA treatment available in China. It can improve the motor, respiratory, digestive, and other system functions in patients [44-47]. Because the sample size of patients with Nusinersen treatment was very small, we suggested to interpret our finding with caution and we recommended further studies to confirm or reject our finding.

We observed that the QoL scores in our study were lower than those reported in previous studies conducted in developed country yy[22]. The potential explanations were: First, China is a developing country while American/European countries are developed ones. The cost of treatment is prohibitive for most SMA patients in China, and they cannot receive the early diagnosis and treatments obtained by patients of foreign countries. Second, the development of diagnosis and treatment of SMA in China is slower than that in foreign countries, and drugs appear on the market later than in western countries. Third, the United States and Belgium have incorporated SMA into newborn screening and has attached great importance to the diagnosis and treatment of SMA; whereas China is still in early stages.

Our study has several strengths. To our best knowledge, it was the first study to evaluate QoL of Chinese patients with SMA and to reflect the QoL of these children according to the perceptions of the caregivers by reliable and valid instruments. Additionally, we have assessed a comprehensive set of potential associated factors of QoL, including SMA subtypes, disease-related characteristics and clinical treatments. Previous studies have not provided evidence on the QoL of patients with SMA in China, and few studies exist regarding the influence of clinical treatments on QoL of patients and their caregivers. Given that China is different from developed countries in social, economic, and medical characteristics, it is necessary and important to measure QoL of SMA and their caregivers in China, as well as the determinants of QoL.

Our study had several limitations worthy to mention. First, the sample size of this study is moderate, reflecting the rarity of the disease. We have not applied self-report in our study because the sample size would be even smaller if we only included children older than 5 years. In the future study, we recommended further studies with larger samples could apply both self-reported and proxyreported QOL measurements among patients with SMA to get a full picture of patient's QOL. A second limitation of this study is that patients were only sampled from central and east China. Given the lack of QoL surveys on SMA patients in other regions, the study may not be representative of SMA patients across China. Nevertheless, our results may be generalized to patients from the areas mentioned above. Third, this study was a crosssectional study with data collected at a single time point, which precluded drawing conclusions regarding causality. Fourth, owing to less data of specific items of clinical treatments, our study cannot provide a more in-depth analysis; so future research is needed with a larger sample that includes subsets of clinical treatments. In addition, our study reflected the influence of disease-related characteristics on the QoL of children and only the initial effect of medical measures. Finally, although the PedsQL is designed to be used for all types of diseases, this survey instrument has not been specifically validated for proxyreported use in children with SMA.

In summary, further follow-ups of these patients should be conducted and QoL indicators should be used to investigate the effectiveness of clinical treatments for childhood SMA. In addition, future studies need larger sample sizes and should pursue multicenter enrollments to involve patients with SMA from all of China to generate a database that reflects the QoL of patients across the country. To confirm the effectiveness of treatment approaches and to make the results more generalizable, subsequent studies should continue research in the diagnosis and treatment of SMA, and should include larger numbers of patients, especially in the drug treatment group.

\section{Conclusion}

To conclude, the more severe the SMA disorder was, the lower were the average scores on the PedsQL NMM and PedsQL FIM as reported by the patients' caregivers, and the poorer was the patients' QoL. Disease-related clinical features and clinical treatments have a significant influence on the QoL of patients with SMA. We recommend implementing clinical treatments, supplemented by exercise training and respiratory support, regularly assessing the functional status of various systems, and establishing MDT health management in patients as early as possible, which can effectively prevent or delay the emergence of disease-related clinical characteristics, can improve patients' QoL, and can relieve caregivers' psychological pressures and overall life burden.

\section{Supplementary Information}

The online version contains supplementary material available at https://doi. org/10.1186/s13023-020-01638-8.

Additional file 1. Detailed dimension of proxy-report of PedsQL ${ }^{\mathrm{TM}} \mathrm{Neuro-}$ muscular Module and PedsQL ${ }^{\mathrm{TM}}$ Family Impact Module. 


\section{Abbreviations}

SMA: Spinal muscular atrophy; QoL: Quality of life; SMN1: Survival motor neuron 1; SMN: Survival of motor neuron; PedsQL NMM: Pediatric Quality of Life Inventory 3.0 Neuromuscular Module; PedsQL FIM: Pediatric Quality of Life Inventory Family Impact Module; MDT: Multidisciplinary team; SD: Standard deviation; SOC: Standard of care.

\section{Acknowledgements}

We thanks all the patients and families for their contribution to this work. We also thank Mingjuan Jin for the contribution on the statistic methods, thank Bin Ma and Huanping Xing for the support of this work and thank Bob for the polish on the language in our paper.

\section{Authors' contributions}

MY, YM, SSM and YX designed the study questionnaire with input from the other authors. MY and SSM coordinated ethics application. YM managed the acquisition of data. MY, YM and RYQ analyzed data and interpreted findings with input from the other authors. MY drafted the manuscript. CZY and GNB substantially revised and edited the manuscript. All authors reviewed the final manuscript and approved the decision to submit for publication. MY and YM as first authors and SSM and GNB as last authors equally contributed to this manuscript. All authors read and approved the final manuscript.

\section{Funding}

This work is supported by National Natural Science Foundation (81801490 \& 81741076) and Zhejiang Province Public Welfare Technology Application Research Project (LGC21H090001).

\section{Availability of data and materials}

All data generated during this study are included in this published article.

\section{Ethics approval and consent to participate}

This study was approved by Ethical Committee of Children's Hospital, Zhejiang University School of Medicine (2019-IRB-171). Informed consent was obtained from all participants before the study questionnaires were completed.

\section{Consent for publication}

The consent for publication has been obtained from all authors.

\section{Competing interests}

No benefits in any form have been received or will be received from a commercial party related directly or indirectly to the subject of this article.

\section{Author details \\ ${ }^{1}$ Department of Neurology, Children's Hospital, Zhejiang University School of Medicine, National Clinical Research Center for Child Health, Hang- zhou 310052, China. ${ }^{2}$ School Public Health of Zhejiang University, Children's Hospital, Zhejiang University School of Medicine, National Clinical Research Center for Child Health, Hangzhou 310052, China.}

Received: 27 July 2020 Accepted: 3 December 2020 Published online: 06 January 2021

\section{References}

1. Farrar MA, Park SB, Vucic S, Carey KA, Turner BJ, Gillingwater TH, et al. Emerging therapies and challenges in spinal muscular atrophy. Ann Neurol. 2017:81:355-68.

2. Groen EJN, Talbot K, Gillingwater TH. Advances in therapy for spinal muscular atrophy: promises and challenges. Nat Rev Neurol. 2018;14:214-24.

3. Schmalbruch $\mathrm{H}$, Haase $\mathrm{G}$. Spinal muscular atrophy: present state. Brain Pathol. 2001;11:231-47.

4. Ahmad S, Bhatia K, Kannan A, Gangwan L. Molecular mechanisms of neurodegeneration in spinal muscular atrophy. J Exp Neurosci. 2016:10:39-49.

5. Oskoui M, Kaufmann P. Spinal muscular atrophy. Neurotherapeutics. 2008:5:499-506

6. Russman BS. Spinal muscular atrophy: clinical classification and disease heterogeneity. J Child Neurol. 2016:22:946-51.
7. Mercuri E, Bertini E, lannaccone ST. Childhood spinal muscular atrophy: controversies and challenges. Lancet Neurol. 2012;11:443-52.

8. Faravelli I, Nizzardo M, Comi GP, Corti S. Spinal muscular atrophyrecent therapeutic advances for an old challenge. Nat Rev Neurol. 2015;11:351-9.

9. Cynthia CJ, Suzanne FC, Jill J, Lisa B, Sandra PR, John S, et al. Spinal muscular atrophy (SMA) subtype concordance in siblings: findings from the cure SMA cohort. J Neuromuscul Dis. 2020;7:33-40.

10. Shababi M, Lorson CL, Rudnik Schöneborn SS. Spinal muscular atrophy: a motor neuron disorder or a multi-organ disease? J Anat. 2014;224:15-28.

11. Hamilton G, Gillingwater TH. Spinal muscular atrophy: going beyond the motor neuron. Trends Mol Med. 2013;19:40-50.

12. Nash LA, Burns JK, Chardon JW, Kothary R, Parks RJ. Spinal muscular atrophy: more than a disease of motor neurons? Curr Mol Med. 2016;16:779-92.

13. Finkel RS, Mercuri E, Meyer OH, Simonds AK, Schroth MK, Graham RJ, et al. Diagnosis and management of spinal muscular atrophy: Part 2: pulmonary and acute care; medications, supplements and immunizations; other organ systems; and ethics. Neuromuscul Disord. 2018;28:197-207.

14. Mercuri E, Finkel RS, Muntoni F, Wirth B, Montes J, Main M, et al. Diagnosis and management of spinal muscular atrophy: Part 1: recommendations for diagnosis, rehabilitation, orthopedic and nutritional care. Neuromuscul Disord. 2018;28:103-15.

15. Grychtol R, Abel F, Fitzgerald DA. The role of sleep diagnostics and noninvasive ventilation in children with spinal muscular atrophy. Paediatr Respir Rev. 2018;28:18-25.

16. Takei S, Miyagi M, Saito W, Imura T, Inoue G, Nakazawa T, et al. Safety and efficacy of treatment for scolios is secondary to spinal muscular atrophy fused to lumbar 5 level. Spine Surg Relat Res. 2018;2:294-8.

17. Mehta NM, Newman H, Tarrant S, Graham RJ. Nutritional status and nutrient intake challenges in children with spinal muscular atrophy. Pediatr Neurol. 2016;57:80-3.

18. Martinez MP. What is quality of life and how do we measure it? Relevance to Parkinson's disease and movement disorders. Mov Disord. 2017;32:382-92.

19. Vanleerberghe P, De Witte N, Claes C, Schalock RL, Verté D. The quality of life of older people aging in place: a literature review. Qual Life Res. 2017:26:2899-907.

20. Haraldstad K, Wahl A, Andenaes R, Andersen JR, Andersen MH, Beisland $E$, et al. A systematic review of quality of life research in medicine and health sciences. Qual Life Res. 2019;28:2641-50.

21. Rouault F, Christie-Brown V, Broekgaarden R, Gusset N, Henderson D, Marczuk $P$, et al. Disease impact on general well-being and therapeutic expectations of European Type II and Type III spinal muscular atrophy patients. Neuromuscul Disord. 2017;27:428-38.

22. Weaver MS, Hanna R, Hetzel S, Patterson K, Yuroff A, Sund S, et al. A prospective, crossover survey study of child- and proxy-reported quality of life according to spinal muscular atrophy type and medical interventions. J Child Neurol. 2020;35:322-30.

23. Hu J, Jiang L, Hong SQ, Cheng L, Kong M, Ye YZ. Reliability and validity for Chinese version of pediatric quality of life inventory TM(PedsQLTM) 30 neuromuscular module. J Chongqing Med Univ. 2012;37:806-10.

24. Chen RQ, Hao YT, Feng LF, Zhang YF, Huang ZY. The Chinese version of the Pediatric Quality of Life Inventory ${ }^{\mathrm{TM}}$ (PedsQL $^{\mathrm{TM}}$ ) Family Impact Module: cross-cultural adaptation and psychometric evaluation. Health Quality Life Outcomes. 2011;9:16.

25. Cohen J. Statistical power analysis for the behavioral sciences. 2nd ed. Hillsdale: L. Erlbaum; 1988

26. Min R, Linli P, Qin L, Michelle P, Fang H, Hong W. The association between quality of life (QoL) and health literacy among junior middle school students: a cross-sectional study. BMC Public Health. 2018;18:1183.

27. Vai S, Bianchi ML, Moroni I, Mastella C, Broggi F, Morandi L, et al. Bone and spinal muscular atrophy. Bone. 2015;79:116-20.

28. Merlini L, Granata C, Bonfiglioli S, Marini ML, Cervellati S, Savini R. Scoliosis in spinal muscular atrophy: natural history and management. Dev Med Child Neurol. 1989;31:501-8.

29. Cremers CH, Fischer MJ, Kruitwagen-van Reenen ET, Wadman RI, Vervoordeldonk JJ, Verhoef M. Participation and mental well-being of mothers of home-living patients with spinal muscular atrophy. Neuromuscul Disord. 2019:29:321-9. 
30. Veilleux L, Rauch F. Muscle-bone interactions in pediatric bone diseases. Curr Osteoporos Rep. 2017;15:425-32.

31. Coffey VG, Hawley JA. The molecular bases of training adaptation. Sports Med. 2007;37:737-63.

32. Motl RW, Sandroff BM. Benefits of exercise training in multiple sclerosis. Curr Neurol Neurosci Rep. 2015;15:1-9.

33. Ng S, Manta A, Ljubicic V. Exercise biology of neuromuscular disorders. Appl Physiol Nutr Metab. 2018;43:1194-206.

34. Case LE, Apkon SD, Eagle M, Gulyas A, Juel L, Matthews D, et al. Exercise management of the patient with duchenne muscular dystrophy. Pediatrics (Evanston). 2018;142(Suppl 2):S17-33.

35. Voet NB, van der Kooi EL, van Engelen BG, Geurts AC. Strength training and aerobic exercise training for muscle disease. Rev Cochrane Database Syst Rev. 2019;12:CD003907.

36. Mehta P, Melikishvili A, Carvalho KS. Neurological complications of respiratory disease. Semin Pediatr Neurol. 2017;24:14-24.

37. Boentert M, Wenninger S, Sansone VA. Respiratory involvement in neuromuscular disorders. Curr Opin Neurol. 2017;30:529-37.

38. Von Kodolitsch Y, Rybczynski M, Vogler M, Mir T, Schüler H, Kutsche K, et al. The role of the multidisciplinary health care team in the management of patients with Marfan syndrome. J Multidiscip Healthc. 2016;9:587-614

39. Brook J, McGraw C. Multidisciplinary perspectives: application of the consolidated framework for implementation research to evaluate a health coaching initiative. Health Soc Care Community. 2018;26:e386-95.

40. Auvin S, Bissler JJ, Cottin V, Fujimoto A, Hofbauer G, Jansen AC, et al. A step-wise approach for establishing a multidisciplinary team for the management of tuberous sclerosis complex: a Delphi consensus report. Orphanet J Rare Dis. 2019;14:91.

41. Duis J, van Wattum PJ, Scheimann A, Salehi P, Brokamp E, Fairbrother L, et al. A multidisciplinary approach to the clinical management of PraderWilli syndrome. Mol Genet Genomic Med. 2019;7:e514.

42. Abu-El-Haija M, Nathan JD. Pediatric chronic pancreatitis: updates in the 21st century. Pancreatology. 2018;18:354-9.

43. Chan DS, Callahan CW, Moreno C. Multidisciplinary education and management program for children with asthma. Am J Health Syst Pharm. 2001;58:1413-7.

44. Corey DR. Nusinersen, an antisense oligonucleotide drug for spinal muscular atrophy. Nat Neurosci. 2017;20:497-9.

45. Michelson D, Ciafaloni E, Ashwal S, Lewis E, Narayanaswami P, Oskoui $M$, et al. Evidence in focus: Nusinersen use in spinal muscular atrophy. Neurology. 2018;20:923-33.

46. Darras BT, Farrar MA, Mercuri E, Finkel RS, Foster R, Hughes SG, et al. An integrated safety analysis of infants and children with symptomatic spinal muscular atrophy (SMA) treated with Nusinersen in seven clinical trials. CNS Drugs. 2019;33:919-32.

47. Pane M, Palermo C, Messina S, Sansone VA, Bruno C, Catteruccia M, et al. Nusinersen in type 1 SMA infants, children and young adults: preliminary results on motor function. Neuromuscul Disord. 2018;28:582-5.

\section{Publisher's Note}

Springer Nature remains neutral with regard to jurisdictional claims in published maps and institutional affiliations.
Ready to submit your research? Choose BMC and benefit from:

- fast, convenient online submission

- thorough peer review by experienced researchers in your field

- rapid publication on acceptance

- support for research data, including large and complex data types

- gold Open Access which fosters wider collaboration and increased citations

- maximum visibility for your research: over 100M website views per year

At BMC, research is always in progress.

Learn more biomedcentral.com/submissions 